\title{
Prevalência de comportamentos de risco para transtornos alimentares e uso de dieta "low-carb" em estudantes universitários
}

\author{
Prevalence of eating disorders risk behavior and \\ "low-carb" diet in university students \\ Jônatas de Oliveira' \\ https://orcid.org/0000-0003-2110-5920 \\ Leandro Figueredo ${ }^{2}$ \\ https://orcid.org/0000-0001-6027-1572 \\ Táki Athanássios Cordás ${ }^{1,3}$ \\ https://orcid.org/0000-0003-3929-0175
}

\section{Palavras-chave \\ Transtornos alimentares, bulimia nervosa, transtorno de compulsão alimentar, dieta low-carb.}

\section{RESUMO}

Objetivos: Identificar a presença de compulsão alimentar associada ou não a práticas compensatórias em praticantes de dieta low-carb. Métodos: Foram utilizadas a Escala de Compulsão Alimentar Periódica (ECAP) e o Questionário de Hay para avaliar a frequência de compulsão alimentar e práticas compensatórias, além de um questionário de frequência do consumo de chocolate, pão e arroz. A intensidade de restrição de carboidratos foi avaliada com escala de 1 a 8 pontos. Os participantes foram divididos nos grupos (i) dieta low-carb e (ii) controle. Foram realizadas comparações entre grupos, além de correlações entre variáveis de interesse no grupo dieta ( $p<0,05)$. Resultados: Participaram 853 universitários, sendo 75,97\% muIheres com média de 22,04 anos (DP = 3,33) e índice de massa corporal (IMC) com média de $23,56 \mathrm{~kg} / \mathrm{m}^{2}$ ( $\left.\mathrm{DP}=4,38\right)$. Do total, 214 fizeram dieta low-carb e 639 não. A prevalência de um alto escore sugestivo de compulsão alimentar sem práticas compensatórias foi de 17,94\% (n = 153) e a presença de compulsão alimentar associada com compensação foi de 2,23\% ( $n=19)$. No grupo dieta, 35,05\% ( $n=75)$ também realizaram jejum intermitente. O grupo dieta atingiu maiores valores para ECAP e IMC e menores para frequência de consumo de arroz e pão francês. Ainda, os escores da ECAP se correlacionaram positivamente com o consumo de chocolate $(r=+0,14 ; p=0,0377)$ e valores de IMC $(r=+0,19 ; p=0,0042)$, enquanto a restrição de carboidratos apresentou correlação negativa com o consumo de chocolate $(r=-0,13 ; p=0,041)$, pão francês $(r=-0,20 ; p=0,0024)$ e arroz $(r=-0,36 ; p=<0,0001)$. Conclusões: Destacam-se a alta prevalência da prática de dieta $(25,09 \%)$ e os maiores níveis de compulsão alimentar neste grupo, além de menor consumo de arroz e pão francês em relação aos que não fizeram dieta.

\section{ABSTRACT}

Objectives: To identify the presence of binge eating associated or not with compensatory practices in low-carb dieters. Methods: Binge Eating Scale (BES) and Hay Questionnaire

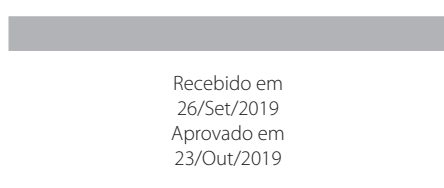

DOI: $10.1590 / 0047-2085000000245$
1 Universidade de São Paulo (USP), Faculdade de Medicina, Hospital das Clínicas, Instituto de Psiquiatria (IPq), Programa de Transtornos Alimentares (AMBULIM/PROTAD), São Paulo, SP, Brasil.

2 Beneficência Portuguesa de São Paulo, São Paulo, SP, Brasil.

3 Universidade de São Paulo (USP), Instituto de Psicologia, São Paulo, SP, Brasil. 


\section{Keywords}

Eating disorders, bulimia nervosa, binge eating disorder, low-carb diet. were used in order to assess the frequency of binge eating and compensatory practices, in addition to a frequency questionnaire for the consumption of chocolate, bread and rice. A scale of 1-8 points assessed the carbohydrate restriction intensity, and participants were divided into groups (i) low-carb diet and (ii) control. Comparisons between groups were part of the analysis, as well as correlations between variables of interest per diet group ( $p<0.05)$.

Results: Participants were a total of 853 university students, in which $75.97 \%$ were women with an average of 22.04 years old $(S D=3.33)$ and an average $B M l$ of $23.56 \mathrm{~kg} / \mathrm{m}^{2}$, (SD = 4.38). From the aforementioned total, 214 had a low-carb diet, and 639 did not. The prevalence of a high score suggestive of binge eating without compensatory practices was 17.94\% ( $n=153)$, while the presence of binge eating associated with compensation was $2.23 \%(n=19)$. As for the diet group, 35.05\% $(n=75)$ also performed intermittent fasting. The diet group reached higher values for ECAP and BMI, and lower for frequency of consumption of rice and bread. Furthermore, ECAP scores correlated positively with chocolate consumption $(r=+0.14$; $p=0.0377)$ and $B M I$ values $(r=+0.19 ; p=0.0042)$, whereas carbohydrate restriction showed negative correlation with chocolate consumption ( $r=-0.13 ; p=0.041)$; French bread $(r=-0.20 ; p=0.0024)$ and rice $(r=-0.36 ; p=<0.0001)$. Conclusions: We highlight the high prevalence of diet practice (25.09\%), and the higher levels of binge eating in this group, as well as the lower consumption of rice and bread compared to those who did not diet.

\section{INTRODUÇÃO}

O aumento do sofrimento psíquico em estudantes universitários, assim como o desenvolvimento de transtornos mentais e casos de suicídio, representam um grande desafio para as políticas internas das instituições e suas ações preventivas e terapêuticas'. Os transtornos alimentares (TA) também fazem parte dessa discussão, considerando que essa população apresenta risco elevado para o desenvolvimento do quadro psiquiátrico 2,3 , mas também desfechos de saúde negativos, como o comprometimento do rendimento escolar, isolamento social e agravo em parâmetros clínicos que alguns comportamentos de risco para TA podem trazer'. Sabe-se que a exposição da mídia, a pressão para atingir um corpo magro e a internalização de um ideal de corpo são preditores do aumento de comportamentos de risco para TA em mulheres ${ }^{5}$ e que eles podem ocorrer como forma de lidar com emoções negativas, cobranças e insatisfação corporal.

Justifica-se então a identificação de uma série de comportamentos de risco, como as dietas restritivas, práticas de jejuns, métodos compensatórios inapropriados (vômitos, uso de laxantes e/ou diuréticos) e atividade física excessiva². Considerando o conjunto dessas práticas, em 2006 a Associação de Psiquiatria Americana propôs o uso do termo "comer transtornado" para descrever o espectro de questões alimentares disfuncionais que vão desde a simples dieta até os TA ${ }^{6}$.

A prevalência de comportamentos de risco para os TA em universitários é descrita principalmente por meio do uso de instrumentos de autorrelato sob a forma de escalas como, por exemplo, o Eating Attitudes Test (EAT-26), sendo o mais utilizado no Brasil ${ }^{3}$, que gera uma série de nomenclaturas distintas como "comportamento de risco para TA", "risco de TA", "suspeita de TA" e "atitudes alimentares negativas", como revisado por Leal et al.".
Foi demonstrado que, segundo o EAT-26, o comportamento de risco para TA variou de $23,7 \%$ a $30,1 \%$ nas cinco regiões do país?. Em outro estudo, foi descrito que 40,7\% dos universitários faziam regime para emagrecer, 35,6\% usavam métodos compensatórios, 23,9\% pulavam refeições, 12,6\% consumiam apenas líquidos ou ficavam sem comer para emagrecer e 3,3\% induziam vômitos ${ }^{8}$.

Definida como a "restrição intencional e sustentada do consumo energético com o propósito de perda de peso ou manutenção", a restrição alimentar está também associada com a "privação hedônica", pois a maioria das dietas restritivas requer uma alimentação com baixo teor de gordura, baixo valor energético total, limitado tamanho e número de porções alimentares e proibição do consumo dos alimentos mais palatáveis ${ }^{10}$. A prática de dietas restritivas é considerada um fator de risco para o desenvolvimento de TA ${ }^{5}$, contudo as dietas antigamente denominadas "Dieta do Dr. Atkins" ou "dieta da proteína" voltaram a ser consideradas para a perda de peso $0^{11-14}$, com algumas modificações, e agora nomeadas de "dieta low-carb" (LC).

A LC consiste, em termos gerais, em uma baixa proporção de carboidratos, que pode variar de 30 a $130 \mathrm{~g}$ de carboidratos por dia, em alguns casos sem restrições de calorias, gordura e proteínas ${ }^{15}$. Porém, alguns estudos a modificaram utilizando diversas abordagens, como visto na LC mediterrânea ${ }^{16}$ e na very $L C$ diet/ketogenic ${ }^{17}$. No Brasil, foi proposta recentemente a quantidade de $80 \mathrm{~g}$ de carboidratos ao dia sem restrições lipídica e proteica. E nesse mesmo estudo se considerou, como prática de jejum, o protocolo de "Jejum Intermitente de 4:3", sendo quatro dias da semana de alimentação ad libitum e três dias de jejum de 16 horas. ${ }^{18}$

As dificuldades para a adesão a dietas restritivas podem ser analisadas no âmbito de (1) comportamentos, motivação e nível de restrição necessários ${ }^{9,10}$, mas também (2) de efeitos 
provenientes da composição da dieta, por exemplo, os desejos intensos por comida que surgem pela fome ${ }^{19,20}$. Ainda, a divulgação e a prática não supervisionada de algumas dietas restritivas estão atreladas com uma falta de conhecimento sobre adequação nutricional em indivíduos da população em geral, e alguns estudos descrevem fatores como uma mentalidade de restrição direcionada aos carboidratos ${ }^{19,20}$.

No Brasil, não existem estudos que tenham verificado a presença de comportamentos de risco para TA associados com a restrição de carboidratos na população universitária, portanto o objetivo primário desse estudo foi analisar se indivíduos que fizeram o uso da LC apresentam CA e presença de práticas compensatórias inapropriadas. Os objetivos secundários foram avaliar: (i) se o consumo de alguns alimentos fonte de carboidratos é menor no grupo que fez LC; (ii) se existe correlação entre a restrição de carboidratos e a frequência de consumo de alimentos.

\section{MÉTODOS}

Trata-se de um estudo transversal que avaliou alunos da Universidade de São Paulo (USP). Foram elegíveis estudantes da graduação ou pós-graduação de ambos os sexos com idade entre 17 e 60 anos, que estivessem de acordo com o Termo de Compromisso Livre e Esclarecido antes do preenchimento do questionário on-line (nº de aprovação FMUSP: 2.695.532). Todas as informações foram autorrelatadas, seja por meio de questões estruturadas especificamente para este estudo (dados demográficos e informações gerais) ou questionários. 0 recrutamento ocorreu entre os meses de 07/2018 e 03/2019, com diversas ações realizadas em redes sociais (divulgação em grupos específicos no Facebook'). Também foram realizadas divulgações por meio de e-mail institucional de alguns programas de graduação e pós-graduação. Aqueles que respondiam ao questionário também recebiam um pedido de divulgação para distribuição. Foram removidos das análises aqueles que assinalaram a opção "não aluno USP" e também os graduandos em Nutrição, pois já existem evidências suficientes sobre o risco para TA nessa população ${ }^{3,21}$.

\section{Avaliação da prática de low-carb e jejum intermitente (J)}

Foram desenvolvidas sete questões (uma adaptada da versão original de uma escala) para investigar a ocorrência e a frequência de uso de LC e também de J:

1) Nos últimos três meses você tentou seguir uma dieta low-carb evitando alimentos fonte de carboidratos? ("sim"; "não"; "não sei o que é dieta low-carb")

2) Nos últimos três meses quanto tempo você ficou em dieta low-carb? ("menos de uma semana", "uma semana", "duas semanas" e "um mês")
3) “Em uma escala de 1 a 8, onde 1 significa nenhuma restrição de carboidratos e 8 significa restrição total de carboidratos, qual número você daria para si mesmo?"

4) Depois que você começou a dieta low-carb, ficou culpado depois de comer algum alimento rico em carboidrato? ("sim", "não" e "não se aplica")

5) Depois que você começou a dieta low-carb, ficou mais preocupado com seu peso e forma corporal? ("sim", "não" e "não se aplica")

6) Nos últimos três meses com qual frequência você consumiu (chocolate, pão e arroz)

7) Nos últimos três meses você fez jejum intermitente? ("sim", "não", "não sei o que é jejum intermitente")

As questões anteriormente descritas foram elaboradas especificamente para esta pesquisa, com exceção da questão \#3, que resulta de uma adaptação realizada na questão \#18 da subescala de restrição cognitiva do questionário The Three Factor Eating ${ }^{22}$, em que se trocou restrição alimentar por restrição de carboidratos. Todas as questões utilizadas seguiram o modelo proposto pelo questionário de Hay, que avalia a frequência de comportamentos de compulsão e práticas compensatórias nos últimos três meses. Para estimar a frequência do consumo de chocolate, pão e arroz, foi utilizado um questionário de frequência alimentar adaptado desenvolvido e validado no Brasil ${ }^{23}$.

\section{Compulsão alimentar e práticas compensatórias inapropriadas}

Para identificar a presença de CA e práticas compensatórias inapropriadas, foram utilizadas a Escala de Compulsão Alimentar Periódica (ECAP) ${ }^{24}$, que possui boa sensibilidade e consistência interna de 0,89 (a de Cronbach), e também o Questionário de Hay ${ }^{25}$ traduzido e validado para o português brasileiro ${ }^{26}$, para avaliar frequência de comportamentos de CA e práticas compensatórias nos últimos três meses. A frequência de CA e práticas compensatórias, segundo o questionário de Hay, é: "nenhuma vez", "menos que 1 vez por semana"; "1 vez por semana" e "duas ou mais vezes por semana". Foram considerados dois critérios para identificar indivíduos com presença de compulsão alimentar associada ou não com práticas compensatórias inapropriadas: (I) pontuação > 17 na ECAP indicando presença de CA ${ }^{24}$ e (II) presença de práticas compensatórias segundo o questionário de Hay (ao menos uma vez por semana nos últimos três meses). Esses critérios foram adotados em referência aos diagnósticos de bulimia nervosa (BN) e transtorno de compulsão alimentar (TCA), segundo o Manual Diagnóstico e Estatístico de Transtornos Mentais (DSM-5) ${ }^{27}$. Assim, para indicar casos sugestivos aos quadros, deveriam ser atendidos os critérios I e || para indicativos de BN, e apenas o critério I nos casos sugestivos para TCA. Considerando o caráter não diagnóstico desses instrumentos, utilizaram-se os termos "CA sem 
práticas compensatórias", em relação aos casos sugestivos para TCA, e o termo "CA com práticas compensatórias", em relação aos casos sugestivos para BN.

\section{Análise de dados}

Os dados foram expressos em média e desvio-padrão (DP), e para comparações entre grupos praticante ou não de dieta, foi utilizado o teste de Mann-Whitney, devido à distribuição não normal (verificado após o teste de Shapiro-Wilk). Foram realizadas análises de correlação de Spearman entre variáveis de interesse no grupo dieta e considerou-se o nível de significância de 5\% (GraphPad Prism 7.0).

\section{RESULTADOS}

A maioria dos participantes foram mulheres $(75,97 \% ; n=$ 648). A idade foi de 22,04 anos em média ( $D P=3,33$; mín: 17 e máx: 51 ) e o IMC foi em média de $23,56 \mathrm{~kg} / \mathrm{m}^{2}$ (DP = 4,38; mín: 14 e máx: 42; n = 846; 7 pessoas não sabiam relatar o peso). Os estudantes se dividiram nas áreas de formação acadêmica: Biológicas $(43,85 \%, n=374)$, seguido dos cursos da área de Humanas $(34 \%, n=290)$ e em menor proporção da área de Exatas $(11,96 \%, n=102)$. Também houve participantes representantes da Pós-Graduação (10,20\%, n = 87). A prevalência de busca de tratamento para os três principais TA foi de 4,34\% para TCA, seguida de 1,06\% para BN e 1,99\% para anorexia nervosa.

\section{Dieta low-carb}

Sobre a prática de LC, 5,16\% ( $n=44)$ declararam não conhecer a dieta, enquanto $25,09 \%(n=214)$ realizaram a dieta e $69,40 \%$ ( $n=592)$ não. Nas comparações com o grupo controle $(n=639)$, os indivíduos que realizaram a dieta $(n=214)$ apresentaram maiores valores para CA, peso e IMC e menores valores para frequência de consumo de arroz e pão francês (Tabela 1). A questão "Em uma escala de $1 \boldsymbol{a} \mathbf{8}$, onde 1 significa nenhuma restrição de carboidratos e 8 significa restrição total de carboidratos, qual número você daria para si mesmo?" apresentou correlações negativas com o consumo de chocolate $(r=-0,13 ; p=0,041)$, pão francês $(r=-0,20 ; p=0,0024)$ e arroz $(r=-0,36 ; p=<0,0001)$.

Tabela 1. Comparações entre praticantes ou não de dieta low-carb

\begin{tabular}{|c|c|c|c|}
\hline & Low-carb $(n=214)$ & Controle $(n=639)$ & \\
\hline & Média (DP) & Média (DP) & Valordep \\
\hline $\mathrm{CA}+$ práticas compensatórias & $n=16 ; 7,48 \%$ & $\mathrm{n}=3 ; 1 \%$ & - \\
\hline CA sem práticas compensatórias & $\mathrm{n}=51 ; 23,83 \%$ & $n=102 ; 16 \%$ & - \\
\hline $\operatorname{IMC}\left(\mathbf{k g} / \mathbf{m}^{2}\right)$ & $24,52(4,06)$ & $23,24(4,44)$ & $<0,0001$ \\
\hline Peso (kg) & $67,08(13,08)$ & $65,05(14,98)$ & 0,0035 \\
\hline ECAP & $16,75(9,06)$ & $11,01(7,81)$ & $<0,0001$ \\
\hline \multicolumn{4}{|l|}{ Questionário de Hay - CA } \\
\hline Nenhuma vez & $\mathrm{n}=59 ; 28 \%$ & $n=335 ; 52 \%$ & - \\
\hline Menos que uma vez por semana & $n=90 ; 42 \%$ & $n=193 ; 30 \%$ & - \\
\hline Uma vez por semana & $\mathrm{n}=40 ; 19 \%$ & $n=68 ; 11 \%$ & - \\
\hline Duas ou mais vezes por semana & $n=25 ; 12 \%$ & $\mathrm{n}=43 ; 7 \%$ & - \\
\hline \multicolumn{4}{|c|}{ Questionário de Hay - Práticas purgativas } \\
\hline Nenhuma vez & $\mathrm{n}=158 ; 74 \%$ & $\mathrm{n}=612 ; 94 \%$ & - \\
\hline Menos que uma vez por semana & $n=37 ; 17 \%$ & $\mathrm{n}=23 ; 4 \%$ & - \\
\hline Uma vez por semana & $n=8 ; 4 \%$ & $\mathrm{n}=3 ; 1 \%$ & - \\
\hline Duas ou mais vezes por semana & $\mathrm{n}=11 ; 5 \%$ & $\mathrm{n}=1 ; 1 \%$ & - \\
\hline \multicolumn{4}{|l|}{ Frequência de consumo de alimentos } \\
\hline Chocolate & $2,03(1,45)$ & $1,96(1,49)$ & 0,4189 \\
\hline Pão francês & $1,91(1,66)$ & $2,39(1,67)$ & 0,0013 \\
\hline Arroz & $3,00(2,06)$ & $4,29(1,74)$ & $<0,0001$ \\
\hline \multicolumn{4}{|l|}{ Tempo em dieta low-carb } \\
\hline Menos de uma semana & $\mathrm{n}=65 ; 30,37 \%$ & - & - \\
\hline Uma semana & $\mathrm{n}=41 ; 19,16 \%$ & - & - \\
\hline Duas semanas & $\mathrm{n}=52 ; 24,30 \%$ & - & - \\
\hline Um mês & $n=56 ; 26,17 \%$ & - & - \\
\hline
\end{tabular}

CA: compulsão alimentar; ECAP: Escala de Compulsão Alimentar Periódica. Para dados de IMC/peso: grupo low-carb ( $n=213)$ e grupo controle $(n=633)$. 
No grupo dieta, a prevalência de preocupação com o consumo de carboidratos após o início de LC foi de 77,1\% (n = 165), culpa após comer carboidratos, de 53,74\% ( $n=115)$ e aumento da preocupação com o peso e a forma corporal, de $71,03 \%$ ( $n=152)$. Também, os escores da ECAP se correlacionaram positivamente com a frequência de consumo de chocolate $(r=+0,14 ; p=0,0377)$, peso $(r=+0,17 ; p=0,0121)$ e IMC $(r=+0,19 ; p=0,0042)$

Sobre a prática do Jl, na amostra total, 18,99\% ( $n=162)$ não conheciam, enquanto 20,16\% ( $n=172$ ) jejuaram e 60,84\% $(n=519)$ não. No grupo dieta, 35,05\% $(n=75)$ realizaram LC em associação com Jl.

\section{Comportamento alimentar}

Em relação ao comportamento alimentar, segundo o questionário de Hay, 53,81\% ( $n=459)$ reportaram CA e 9,73\% ( $n=$ 83) reportaram comportamentos compensatórios (Tabela 2).

Tabela 2. Dados gerais dos participantes $(n=853)$

\begin{tabular}{|c|c|c|}
\hline & $\mathrm{n}$ & $\%$ \\
\hline \multicolumn{3}{|l|}{ IMC } \\
\hline Baixo peso & 68 & 7,97 \\
\hline Eutrofia & 531 & 62,25 \\
\hline Sobrepeso & 168 & 19,70 \\
\hline Obesidade grau I & 62 & 7,27 \\
\hline Obesidade graus II e III & 17 & 1,99 \\
\hline \multicolumn{3}{|l|}{ Etnia } \\
\hline Brancos & 652 & 76,44 \\
\hline Pardos e negros & 141 & 16,53 \\
\hline Asiáticos & 45 & 5,74 \\
\hline Indígenas & 4 & 0,47 \\
\hline "Não declarada" & 11 & 1,29 \\
\hline \multicolumn{3}{|l|}{ Frequência de fumo } \\
\hline 1-3 cigarros/dia & 29 & 3,40 \\
\hline 4-5 cigarros/dia & 16 & 1,88 \\
\hline$>10$ cigarros/dia & 11 & 1,29 \\
\hline \multicolumn{3}{|l|}{ Consumo alcoólico } \\
\hline Uma a três vezes por mês & 314 & 36,81 \\
\hline Três vezes por semana & 32 & 3,75 \\
\hline Duas vezes por semana & 77 & 9,03 \\
\hline Uma vez por emana & 127 & 14,89 \\
\hline Uma vez por dia & 2 & 0,23 \\
\hline \multicolumn{3}{|c|}{ Questionário de Hay - Compulsão alimentar } \\
\hline Nenhuma vez & 394 & 46,19 \\
\hline Menos que uma vez por semana & 283 & 33,18 \\
\hline Uma vez por semana & 108 & 12,66 \\
\hline Duas ou mais vezes por semana & 68 & 7,97 \\
\hline \multicolumn{3}{|c|}{ Questionário de Hay - Práticas purgativas } \\
\hline Nenhuma vez & 770 & 90,27 \\
\hline Menos que uma vez por semana & 60 & 7,03 \\
\hline Uma vez por semana & 11 & 1,29 \\
\hline Duas ou mais vezes por semana & 12 & 1,41 \\
\hline
\end{tabular}

Segundo a ECAP, a média da amostra total foi de 12,45 (DP = 8,51; mín-máx = 0 - 46), e de acordo com a pontuação de corte, $24,62 \%(n=210)$ apresentaram CA ( $n=149$ com nível moderado de CA e 61 com nível grave). Na tabela 3 foram separadas 10 afirmativas distintas da ECAP e apresentadas as prevalências de respondentes em ambos os grupos. Destaca-se que $68,82 \%(n=143)$ dos praticantes de LC assinalaram ter dúvida sobre a fome física e 46,32\% ( $n=296$ ) no grupo controle.

\section{Compulsão alimentar associada com práticas compensatórias}

No total avaliado, foram identificados 19 universitários (2,23\% em relação ao total) com presença de CA associada com práticas compensatórias (e, desses, 16 fizeram LC). Eles estavam distribuídos nos cursos: Direito $(n=2)$, Letras ( $n=$ 3), Gestão Ambiental $(n=2)$, e um representante por curso: Engenharia Agronômica, Marketing, Têxtil e Moda, Medicina Veterinária, Farmácia-Bioquímica, Obstetrícia, Economia, Biblioteconomia, Engenharia Civil, História, e apenas aluno de Mestrado. Além disso, um participante assinalou a opção "meu curso não consta na lista".

\section{Compulsão alimentar não associada com práticas compensatórias}

Foram identificados 153 indivíduos (17,94\% em relação ao total) com CA não associada com práticas compensatórias (desses, $n=51$ fizeram LC). Os indivíduos estavam distribuídos nos cursos: Letras ( $n=19$ ), Ciências Biológicas ( $n=$ 13), Farmácia-Bioquímica ( $n=11)$, Direito $(n=8)$, Química

Tabela 3. Questões alimentares presentes em trechos das afirmativas da Escala de Compulsão Alimentar Periódica

\begin{tabular}{|c|c|c|}
\hline Questões & $\operatorname{Dieta}(n=214)$ & Controle (n=639) \\
\hline $\begin{array}{l}\text { \#16 (afirmativa 2) "Me sinto em dúvida para } \\
\text { saber se estou ou não fisicamente com fome" }\end{array}$ & $n=143(68,82 \%)$ & $n=296(46,32 \%)$ \\
\hline $\begin{array}{l}\text { \#7 (afirmativa 2) "Sinto como se tivesse } \\
\text { estragado tudo e como ainda mais" }\end{array}$ & $n=68(31,78 \%)$ & $n=154(24,10 \%)$ \\
\hline $\begin{array}{l}\text { \#15 (afirmativa 3) "Não posso pensar em mais } \\
\text { nada a não ser comida" }\end{array}$ & $n=64(29,91 \%)$ & $n=96(15,02 \%)$ \\
\hline $\begin{array}{l}\text { \#7 (afirmativa 4) "Minha vida parece ser uma } \\
\text { festa ou um morrer de fome" }\end{array}$ & $n=67(31,31 \%)$ & $\mathrm{n}=80(12,52 \%)$ \\
\hline $\begin{array}{l}\text { \#6 (afirmativa 3) "Quase o tempo todo sinto } \\
\text { culpa ou ódio depois de comer demais" }\end{array}$ & $n=56(26,17 \%)$ & $\mathrm{n}=47(7,36 \%)$ \\
\hline $\begin{array}{l}\text { \#8 (afirmativa 4) "Me sinto bastante } \\
\text { desconfortável depois de comer" }\end{array}$ & $n=13(6,07 \%)$ & $n=29(4,54 \%)$ \\
\hline $\begin{array}{l}\text { \#14 (afirmativa 4) "A maior parte das horas } \\
\text { que passo acordado(a) estão pré-ocupadas } \\
\text { por pensamentos sobre comer ou não comer" }\end{array}$ & $n=22(10,28 \%)$ & $n=12(1,88 \%)$ \\
\hline $\begin{array}{l}\text { \#10 (afirmativa 4) "Incapaz de controlar } \\
\text { impulsos" }\end{array}$ & $n=14(6,54 \%)$ & $\mathrm{n}=11(1,72 \%)$ \\
\hline $\begin{array}{l}\text { \#12 (afirmativa 4) "Escolho horas para comer } \\
\text { demais quando sei que ninguém me verá" }\end{array}$ & $n=10(4,67 \%)$ & $n=9(1,41 \%)$ \\
\hline $\begin{array}{l}\text { \#5 (afirmativa 4) "Como o alimento para } \\
\text { satisfazer minha 'fome na boca', em seguida } \\
\text { eu o cuspo, assim não ganharei peso" }\end{array}$ & $n=10(4,67 \%)$ & $n=4(1 \%)$ \\
\hline
\end{tabular}


( $n=7)$, Ciências Biomédicas $(n=7)$, História $(n=6)$, Gestão Ambiental $(n=6)$, Medicina Veterinária $(n=6)$. Foram também identificados quatro indivíduos nos cursos: Matemática, Medicina, Arquitetura e Urbanismo. Três indivíduos em cada um dos cursos: Economia e Controladoria, Obstetrícia e Psicologia. Dois representantes nos cursos: Economia, Enfermagem, Engenharia Florestal, Gerontologia, Administração, Marketing, Publicidade e Propaganda e Terapia Ocupacional. Também, um representante de cada curso: Astronomia, Biblioteconomia, Ciências da Computação, Ciências dos Alimentos, Engenharia Agronômica, Engenharia Ambiental, Engenharia de Produção, Fonoaudiologia, Geofísica, Geografia, Gestão de Políticas Públicas, Informática Biomédica, Jornalismo, Turismo, Meteorologia, Relações Públicas, Saúde Pública e Têxtil e Moda. Representantes da Pós-graduação: Mestrado, $n=12$, Doutorado, $n=1$, e Pós-doutorado, $n=1$. Apenas um participante assinalou a opção "meu curso não consta na lista".

\section{DISCUSSÃO}

Este estudo apresentou a prevalência de sintomas de CA e práticas compensatórias inapropriadas em 853 universitários $(75,97 \%$ mulheres) provenientes em maioria de cursos da área Biológicas (43,85\%). Apesar de metade da amostra ( $n=459)$ ter reportado CA e 9,73\% $(n=83)$ terem reportado comportamentos compensatórios inapropriados, segundo os critérios utilizados para rastreamento de TA, seriam elegíveis para indicativo de TA $20,16 \%$ da amostra $(n=172)$.

O período acadêmico constitui um grande estressor que pode proporcionar o uso da comida como recompensa ou para alívio de sintomas disfóricos. Três situações importante foram elencadas por Nogueira-Martins et al., para o fenômeno estudante-ambiente acadêmico: (1) Adaptação em processos que geram sofrimento, (2) Crises adaptativas durante o desenvolvimento pessoal e (3) Crises psicopatológicas em estudantes que apresentam transtornos mentais'.

No Brasil, apenas dois estudos utilizaram ECAP para rastreamento de TCA ${ }^{28,29}$, e foram encontradas as prevalências de 18\% em estudantes do Rio Grande do Sul (instituição privada $)^{28}$ e de $12,90 \%$ em estudantes do interior de São Paulo (instituição pública) ${ }^{29}$. No último estudo, os escores da ECAP demonstraram valores entre 0 e 36 , enquanto os resultados aqui descritos variam entre 0 e 46 . Nossos dados indicam que 24,62\% dos universitários apresentaram CA segundo a ECAP (desses, 149 com nível moderado e 61 com nível grave). A prevalência de casos de compulsão sem práticas compensatórias foi de 17,93\% ( $n=153)$, enquanto a presença de CA com práticas compensatórias foi identificada em 2,23\% ( $n=19)$.

O grupo que realizou LC $(n=214)$ apresentou maiores valores para IMC, e os escores para ECAP demonstraram correlação positiva com frequência de consumo de chocolate, peso e IMC. Apesar de a frequência de consumo de pão francês e arroz ter sido significativamente menor no grupo que fez LC, não houve diferença para o consumo de chocolate em comparação com o grupo controle $(n=639)$.

O modelo proposto por Papie e Aarts (2013) propõe que a falha em limitar a ingestão de alguns alimentos se dá por influências ambientais (que induzem o consumo alimentar), que atuam em conjunto com uma inibição nos processos de controle $^{19}$, dificultando a perda de peso. No grupo dieta, a restrição direcionada para alimentos que são da rotina da alimentação brasileira e presentes nas refeições principais (pão e arroz) provavelmente contribui para valores similares entre os grupos nas comparações de frequência de consumo de chocolate (2,03 $\pm 1,45$ vs. 1,96 $\pm 1,49$ no grupo controle).

Os resultados da questão adaptada da subescala de restrição cognitiva da The Three Factor Eating, na qual os respondentes indicavam de 1 a 8 o quanto restringiam carboidratos, apresentaram correlações negativas com os escores de frequência de consumo de chocolate ( $p=0,041)$, pão francês ( $p$ $=0,0024)$ e arroz ( $p=<0,0001)$, nos últimos três meses. São necessárias análises futuras sobre a adaptação da escala, sendo esses resultados preliminares.

A restrição alimentar surge, muitas vezes, da insatisfação corporal advinda dos discursos que promovem a prática de restrição dietética. Em muitas sociedades, estar "em dieta" é visto como um comportamento cotidiano e justificado como a busca pela saúde ${ }^{30}$. Enquanto isso, a pressão social que esse conceito gera sugere que os indivíduos que não estão em busca da magreza sejam estigmatizados por suas escolhas individuais e também pelo tamanho de seus corpos. Neste estudo não foram avaliados parâmetros relacionados à imagem corporal e à insatisfação, mas recentemente Kessler e Poll publicaram dados sobre a insatisfação corporal, verificando que em 225 universitários, apesar de mais da metade da amostra $(69,8 \%)$ estar na categoria de eutrofia, $74,7 \%$ gostariam de pesar menos ${ }^{31}$.

É de nosso conhecimento que este é o primeiro estudo que avaliou questões específicas da ECAP, que demonstram uma série de atitudes alimentares relevantes ao contexto de BN e TCA. Na tabela 3, descrevemos 10 afirmativas diferentes das questões da ECAP; originalmente a escala conta com 16 questões, que são divididas entre três ou quatro afirmativas. Sobre a questão \#16, que trata da dúvida em saber se há fome física, 68,82\% dos praticantes de LC a assinalaram, enquanto no grupo que não fez dieta $46,32 \%$ assinalaram a questão. Deve-se considerar que o grupo controle conta com 102 indivíduos com CA sem práticas purgativas, enquanto no grupo dieta há 153 indivíduos, portanto nem todos os indivíduos com CA faziam LC.

Os pensamentos extremistas sobre alimentação, como descrito na questão \#7 "Sinto como se tivesse estragado tudo e como ainda mais", foram presentes em 31,78\% do grupo LC, enquanto no grupo controle, em $24,10 \%$. Nas outras ques- 
tões são tratados alguns comportamentos como cuspir para evitar o ganho de peso, que teve maior prevalência no grupo dieta ( $n=10$ vs. $n=4$ no grupo controle). A preocupação com o controle da ingestão de carboidratos parece refletir também a culpa, como descrito na questão \#6 "Quase o tempo todo sinto culpa ou ódio depois de comer demais", assinalada por $26,17 \%$ do grupo dieta e $7,36 \%$ do grupo controle.

Existem diferentes gatilhos para a CA, mas a restrição alimentar (e dentro dessa categoria, os diversos tipos de dieta) é um fator de risco conhecido para o desenvolvimento de $\mathrm{TAs}^{5}$. O contexto cultural que permeia a prática de restrição de carboidratos autoimposta foi avaliado por Pellerano e Gimenes-Minasse por análise de conteúdo, e os elementos discutidos pelos autores incluíram a reconfiguração das práticas alimentares, sociabilidade e influências das redes sociais ${ }^{20}$.

Embora exista um esforço necessário para o melhor entendimento de como a LC pode contribuir para o tratamento da obesidade, a prática não supervisionada tem sido incentivada na era do "vício em açúcar" e uso do Jl associado com LC. Os mecanismos sugeridos para a ação dessa dieta no emagrecimento seriam a redução do apetite, a redução da lipogênese e o aumento da lipólise e do efeito térmico das proteínas ${ }^{11-14}$. Paradoxalmente, o hábito de fazer dieta é preditor para ganho de peso ${ }^{32-34}$. Os desejos intensos por comida e pensamentos por comida são comuns durante os períodos de dietas ${ }^{35}$. Porém, recentemente foi demonstrado que a $L C$ promoveu redução dos desejos intensos por comida no tratamento de obesidade durante quatro meses ${ }^{17}$, entretanto nossos resultados indicam que a prática não supervisionada está associada com alterações no comportamento alimentar.

Por se tratar de um estudo transversal e o método de coleta por meio do autorrelato dos entrevistados, informações como peso e altura e frequências devem ser interpretadas com cautela. Os resultados provenientes da ECAP e Hay devem ser interpretados com cautela, considerando BN e TCA, na ausência de um diagnóstico formal. Deve-se considerar que a questão utilizada sobre a dieta não necessariamente reflete um baixo consumo alimentar desse macronutriente. Também entendemos que não é a frequência de consumo de carboidratos que determina a dieta em " $\mathrm{LC}$ ", mas a quantidade é o fator determinante, sendo assim necessários futuros estudos com avaliação do consumo alimentar associado com parâmetros comportamentais.

\section{CONCLUSÕES}

A prevalência de comportamentos de risco para TA mantém-se alta entre universitários, e neste estudo destacamos a alta prevalência da prática de LC (25,09\%). Destaca-se que os praticantes de LC apresentaram comportamento alimentar agravado (maior pontuação para CA), além do menor consumo de alimentos fonte de carboidratos em relação aos con- troles. A tentativa de restrição de carboidratos, aliada com atitudes como o medo de engordar, o desejo por emagrecer e a insatisfação corporal, parece contribuir para o comportamento de CA. A intenção em restringir carboidratos parece estar associada com menor consumo alimentar em praticantes de LC.

\section{CONTRIBUIÇÕES INDIVIDUAIS}

Jônatas de Oliveira - Contribuiu na concepção do projeto e análise de dados, interpretação e redação.

Leandro Figueredo - Contribuiu com a concepção do projeto, redação e aprovação final da versão.

Táki Athanássios Cordás - Contribuiu com a interpretação, redação e revisão.

\section{CONFLITO DE INTERESSES}

Não há conflito de interesses ou suporte financeiro a ser declarado.

\section{AGRADECIMENTOS}

Os autores gostariam de agradecer a Profa. Dra. Claudia Ridel Juswiak da Universidade Federal de São Paulo (Unifesp), pelo parecer, a gentileza e as estimadas contribuições realizadas em discussões prévias a este estudo, e também a Dra. Angélica Carreira dos Santos, pelo suporte, revisões e discussões realizadas.

\section{REFERÊNCIAS}

1. Nogueira-Martins LA, Nogueira-Martins MCF. Saúde mental e qualidade de vida de estudantes universitários. Rev Psicol Divers Saúde. 2018;7(3):334-7.

2. Leal GVS, Philippi ST, Polacow VO, Cordás TA, Alvarenga MDS. 0 que é comportamento de risco para transtornos alimentares em adolescentes? J Bras Psiquiatr. 2013;62(1):62-75.

3. Trindade AP, Appolinario JC, Mattos P, Treasure J, Nazar BP. Eating disorder symptoms in Brazilian university students: a systematic review and meta-analysis. Rev Bras Psiquiatr. 2019;41(2):179-87.

4. Costa DG, Carleto CT, Santos VS, Haas VJ, Gonçalves RMDA, Pedrosa LAK. Qualidade de vida e atitudes alimentares de graduandos da área da saúde. Rev Bras Enferm. 2018;71(Suppl 4):1642-9.

5. Culbert KM, Racine SE, Klump KL. Research Review: What we have learned about the causes of eating disorders - A synthesis of sociocultural, psychological, and biological research. J Child Psychol Psychiatry. 2015;56(11):1141-64.

6. American Psychiatric Association. Treatment of patients with eating disorders, third edition. American Psychiatric Association. Am J Psychiatry. 2006;163(7 Suppl):4-54.

7. Alvarenga MDS, Scagliusi FB, Philipp ST. Eating disorders risk behavior in Brazilian female university students. Rev Psiquiatr Clin. 2011;38(1):3-7.

8. Alvarenga MS, Lourenço BH, Philippi ST, Scagliusi FB. Disordered eating among Brazilian female college students. Cad Saude Publica. 2013;29(5):879-88. 
9. Conason A. The Influence of Dieting (Hedonic Deprivation) on Food Intake, How It Can Promote Hedonic Overeating, and Mindful-Eating Interventions. Hedonic Eat How Pleas Food Affect Our Brains Behav. 2015;2012.

10. Lowe MR, Butryn ML. Hedonic hunger: A new dimension of appetite? Physiol Behav. 2007;91(4):432-9.

11. Paoli A, Rubini A, Volek JS, Grimaldi KA. Beyond weight loss: A review of the therapeutic uses of very-low-carbohydrate (ketogenic) diets. Eur J Clin Nutr. 2013;67(8):789-96.

12. Moreno B, Bellido D, Sajoux I, Goday A, Saavedra D, Crujeiras AB, et al. Comparison of a very low-calorie-ketogenic diet with a standard low-calorie diet in the treatment of obesity. Endocrine. 2014;47(3):793-805.

13. Hamdy 0, Tasabehji MW, Elseaidy T, Tomah S, Ashrafzadeh S, Mottalib A. Fat versus carbohydrate-based energy-restricted diets for weight loss in patients with type 2 diabetes. Curr Diab Rep. 2018;18(12).

14. Gershuni VM, Yan SL, Medici V. Nutritional Ketosis for Weight Management and Reversal of Metabolic Syndrome. Curr Nutr Rep. 2018;97-106.

15. Hernandez TL, Sutherland JP, Wolfe P, Allian-Sauer M, Capell WH, Talley ND, et al. Lack of suppression of circulating free fatty acids and hypercholesterolemia during weight loss on a high-fat, low-carbohydrate diet. Am J Clin Nutr. 2010;91(3):578-85.

16. Pérez-Guisado J, Mũoz-Serrano A, Alonso-Moraga Á. Spanish Ketogenic Mediterranean diet: A healthy cardiovascular diet for weight loss. Nutr J. 2008;7(1):1-7.

17. Castro Al, Gomez-Arbelaez D, Crujeiras AB, Granero R, Aguera Z, Jimenez-Murcia S, et al. Effect of a very low-calorie ketogenic diet on food and alcohol cravings, physical and sexual activity, sleep disturbances, and quality of life in obese patients. Nutrients. 2018;10(10). pii: E1348.

18. Vargas AJ, Pessoa LS, Rosa RL. Jejum intermitente e dieta low carb na composição corporal e no comportamento alimentar de mulheres praticantes de atividade física. Rev Bras Nutr Esportiva. 2018:12(72):483-90.

19. Stroebe W, van Koningsbruggen GM, Papies EK, Aarts H. Why most dieters fail but some succeed: a goal conflict model of eating behavior. Psychol Rev. 2013;120(1):110-38

20. Pellerano JA, Gimenes-Minasse MHSG. "Low Carb, High Fat": comensalidade e sociabilidade em tempos de dietas restritivas. DEMETRA Aliment Nutr Saúde. 2015;10(3):493-506.

21. Sampaio HAC, Silva IA, Parente NDA, Carioca AAF. Ambiente familiar erisco de transtorno alimentar entre universitários da área da saúde. DEMETRA Aliment Nutr Saúde. 2019:14(33308):1-15.
22. Natacci $L C$, Ferreir Júnior $M$. The three factor eating questionnaire - R21: Tradução para 0 português e aplicação em mulheres brasileiras. Rev Nutr. 2011;24(3):383-94 .

23. Ribeiro AC, Sávio KEO, Rodrigues MDLCF, Costa THM, Schmitz BDAS. Validação de um questionário de frequência de consumo alimentar para população adulta. Rev Nutr. 2006;19(5):553-62

24. Freitas S, Lopes CS, Coutinho W, Appolinario JC. Tradução e adaptação para o português da Escala de Compulsão Alimentar Periódica. Rev Bras Psiquiatr. 2001;23(4):215-20.

25. Hay P. The epidemiology of eating disorder behaviors: an Australian community-based survey. Int J Eat Disord. 1998;23(4):371-82.

26. Ferreira JES, Veiga GV. Confiabilidade (teste-reteste) de um questionário simplificado para triagem de adolescentes com comportamentos de risco para transtornos alimentares em estudos epidemiológicos. Rev Bras Epidemiol. 2008;11(3):393-401.

27. American Psychiatric Association. Diagnostic and Statistical Manual of Mental Disorders. 5th ed. Washington: American Psychiatric Association; 2013.

28. Vitolo MR, Bortolini GA, Horta RL. Prevalência de compulsão a limentar entre universitárias de diferentes áreas de estudo. Rev Psiquiatr Rio Gd Sul. 2006;28(1):20-6.

29. Nicoli MG, Junior RDRL. Binge Eating Disorder and body image perception among university students. Eat Behav. 2011;12(4):284-8.

30. Miranda VPN, Filgueiras JF, Neves CM, Teixeira PC, Ferreira MEC. Insatisfação corporal em universitários de diferentes áreas de conhecimento TT - Body dissatisfaction in college students of different study areas. J Bras Psiquiatr. 2012;61(1):25-32.

31. Kessler AL, Poll FA. Relação entre imagem corporal, atitudes para transtornos alimentares e estado nutricional em universitárias da área da saúde. J Bras Psiquiatr. 2018;67(2):118-25.

32. Lowe MR, Doshi SD, Katterman SN, Feig EH. Dieting and restrained eating as prospective predictors of weight gain. Front Psychol. 2013;4:577.

33. Goldschmidt AB, Wall MM, Choo THJ, Evans EW, Jelalian E, Larson N, et al. Fifteen-year Weight and Disordered Eating Patterns Among Community-based Adolescents. Am J Prev Med. 2018;54(1):e21-9.

34. Lowe MR, Marti CN, Lesser EL, Stice E. Weight suppression uniquely predicts body fat gain in first-year female college students. Eat Behav. 2019;32(2018):60-4.

35. Polivy J, Coleman J, Herman (P. The effect of deprivation on food cravings and eating behavior in restrained and unrestrained eaters. Int J Eat Disord. 2005;38(4):301-9. 